\title{
Computer vision system for estimating and controlling the weight of glass gobs during their industrial formation process
}

\author{
Antonio Ramón Jiménez Ruiz \\ Eskarne Laizola Loinaz \\ Fernando Morgado Rodríguez \\ Mar Calvache Sánchez \\ Fernando Seco Granja \\ Instituto de Automática Industrial-CSIC \\ Ctra. de Campo del Real, km. 0,2, La Poveda, \\ 28500 Arganda del Rey, Madrid, Spain
}

\begin{abstract}
We present a computer vision system for measuring the weight of gobs during a glass-forming process, and a control strategy to automatically correct any weight deviation from a given set point. The system is based on a reliable gob area estimation using image-processing algorithms. A monochrome CCD high-resolution camera and a photodetector for synchronizing acquisition are used for registering gob images. Assuming that the gob has symmetry of revolution about the vertical axis, the proposed system estimates the weight of gobs with accuracy better than $\pm 0.75 \%$. A learning weight control strategy is proposed based on a proportional-integral (PI)-repetitive control scheme. The weight deviation from a set point is used as a control signal to adjust the glass flow into the feeder. This regulation scheme enables effective weight control, canceling mid- and long-term effects. The tracking error of $\pm 1.5 \%$ means a reduction of $40 \%$ when compared with a traditional PI controller. (c) 2004 SPIE and IS\&T. [DOI: 10.1117/1.1762888]
\end{abstract}

\section{Introduction}

Fabrication of glass is a complex industrial process, where the same production lines are employed in different manufacturing processes. Manual modifications are commonplace and are a time-consuming, intuitive task that is usually done by operators with great experience. An automated inspection and control system is proposed that can manage most routine operations.

In the production of glass dishes, the key component is the feeder that transforms a continuous flow of glass into a discrete sequence of glass drops. The feeder (see Fig. 1, right) consists of a cylinder that can be moved vertically to regulate the gob size, a piston used to push glass out, and a shear mechanism to cut the molten glass to obtain the gobs from which the plates are formed. In the fabrication process, control is done by manually moving the cylinder up and down to change the glass flow. Note that the cylinder rotates periodically with a period that expert operators ad-

Paper 03035 received Mar. 4, 2003; revised manuscript received Oct. 3, 2003; accepted for publication Nov. 21, 2003

$1017-9909 / 2004 / \$ 15.00$ @ 2004 SPIE and IS\&T. just empirically. Gobs fall down over molds, and dishes are shaped by centrifugal forces.

Several factors can cause a deviation of the weight of gobs from a predefined reference value. First, there is a random perturbation caused by the lack of synchronization of mechanical devices such as plungers, pistons, etc. Changes in the spinning direction of the tube inside the feeder are necessary to generate a homogeneous composition of the molten glass and cause significant midterm drifts that are quite periodic and repetitive. We observed that the gob weight oscillates with a period that corresponds exactly with the rotation period of the feeder tube, and can be corrected by changing the vertical position of the feeder tube. Additionally, long-term drifts due to changes in the viscosity of the raw glass material affect the weight along several hours of operation.

All these effects result in glass products of varying weight and diameter. In current production processes, about $10 \%$ of the final goods must be rejected because they do not fulfill quality requirements, causing a decrease of efficiency and productivity.

Different commercial systems control weight, shape, and temperature. ${ }^{1,2}$ Those systems are based on linear cameras and perform image reconstruction based on calculation of the speed of the falling gob. Some of them use a dualfrequency IR pyrometer to measure temperature in different points inside the gob. The weight control strategy is normally based on a proportional-integral-derivative (PID) implemented in a programmable logic controller (PLC), which actuates by adjusting the cylinder height or the plunger position.

We propose a system that uses a high-resolution matrix camera to capture a picture of the gob. This method enables a precise area estimation without the necessity of image reconstruction and gob speed estimation. Instead of using a simple PID for the final control, we developed a digital repetitive controller to predict the rotation changes of the feeder tube. A weight scale parallel to the production line is used to calibrate the system, giving the actual weight of 


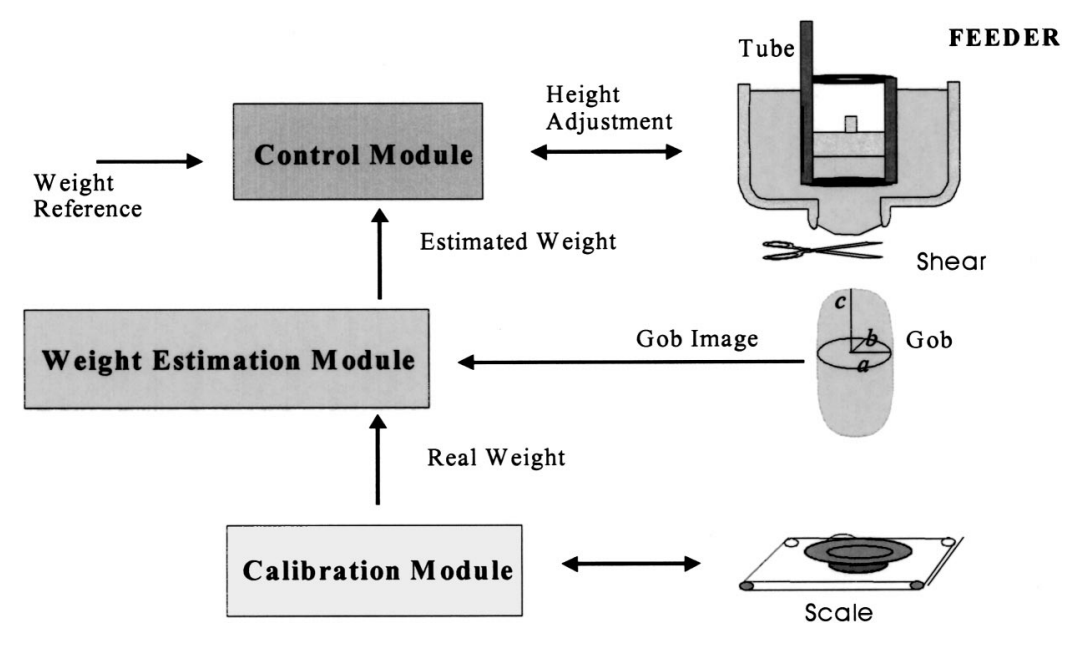

Fig. 1 Gob inspection system.

some dishes taken approximately every $60 \mathrm{~min}$. In the next sections, we give detailed information of the proposed gob inspection system.

\section{Proposed Gob Inspection System: Principle of Operation}

The system we developed can be divided in three different modules that can be studied separately: (1) weight estimation module, (2) control module, and (3) calibration module. Figure 1 shows these modules and the information flow among them when they operate together.

The weight estimation module captures an image and executes an image-processing algorithm that is based on an intensity-level threshold to generate a binary image. Erosion and dilation morphological operators are used to reduce image noise. Region labeling and connection processes are used to distinguish pixels corresponding to the valid gob from other pixels forming significant background residuals that do not belong to the gob area. Once the area is estimated, the volume of the gob is derived, and consequently its weight, assuming a valid glass density value and a calibration value, which is updated once every half hour.

Control is achieved with estimated weight values and the position of the feeder tube. When the estimated weight deviates from the reference, the position of the feeder tube is modified in height via an ac motor. The achieved displacement is measured using a linear variable displacement transformer (LVDT) sensor. As we previously reported, the rotations of the feeder tube produce weight perturbations, but this module uses a learning repetitive control to anticipate the response.

The calibration module measures the real weight of dishes using a precision weight scale in the production line. A calibration constant is updated once an hour by comparing the real weight with weights calculated using the image processing software. This calibration constant integrates parameters that are not precisely known, such as glass density, focal length of the camera lenses, distance from the camera to the gob, and so on, and is used to match estimated weight with real values.

\section{Measurement Subsystem}

The weight measurement estimation can be divided into the following steps: (1) image acquisition, (2) segmentation and image improvement, and, finally, (3) weight estimation. The next subsections provide insight into these points.

\subsection{Image Acquisition}

To select a camera for image acquisition we used the following reasoning. We assume that we can approximate a gob by an ellipsoid with symmetry of revolution around its vertical axis. Assuming a typical gob weight of $410 \mathrm{~g}$ with nearly constant density and a maximum error to be limited by $\pm 2 \mathrm{~g}$, the camera resolution must be at least $640 \times 480$ pixels. To avoid the use of complex subpixel algorithms, ${ }^{3-5}$ which are also more time-consuming, we chose a standard high-resolution 1026×296-pixel camera (JAI CV-M1), which at a $1-\mathrm{m}$ distance from the object plane gives a pixel size of about $0.1 \times 0.1 \mathrm{~mm}$.

When considering the gob as a moving object, it is necessary to take into account the integration or exposure time to use. Neglecting air friction, the velocity of the gob when it is in front of the photodetector is independent of its volume. For a free-falling height of $0.5 \mathrm{~m}$, the velocity of the gob is approximately $3 \mathrm{~m} / \mathrm{s}$. To avoid a vertical displacement of the gob larger than the pixel resolution, that is, 0.1 $\mathrm{mm}$, the exposure time should be less than $1 / 3000 \mathrm{~s}$. This requirement is satisfied by JAI CV-M1 camera, which has an exposure time ranging from 1 to $1 / 10,000 \mathrm{~s}$. The default operating temperature valid for our camera ranges between -5 and $45^{\circ} \mathrm{C}$, so to operate close to the feeder, the camera is cooled using a fan. We chose an optical objective with a relatively long focal length $(f=35 \mathrm{~mm})$ because this type of lens enables acquisition of images with low distortion, and it has no problems with limited depth of field, because distance to the gob is always constant.

To synchronize the gob presence with the camera acquisition trigger, we use a sensor based on a photodetector that generates a transistor-transistor logic (TTL) pulse when it detects an intense source of light. This pulse can be delayed in a range between 10 and $90 \mathrm{~ms}$ to adjust the centering of 

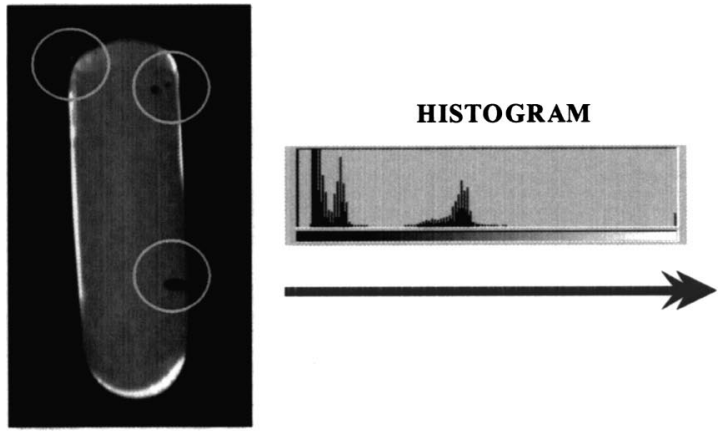

Fig. 2 (Left) intensity image and histogram

(c) dark spots, and (d) boundary holes.

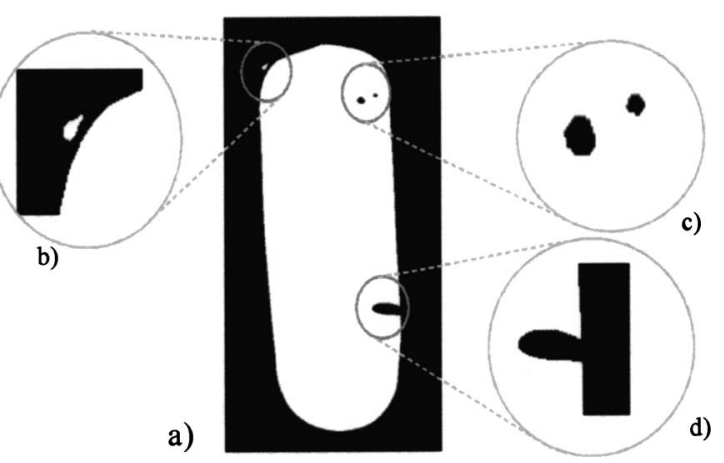

the gob in the registered image. The sensitivity level of the detector can be adjusted to trigger the camera acquisition when enough illumination reaches the sensor. A black cylindrical head is attached in front of the photodiode to allow entry of only light coming along the cylindrical major axis of the photodiode. Therefore, light coming from any direction other than this axis is blocked, thereby eliminating additional light sources in the surrounding working space that could cause false triggers.

We also tested the acquisition of two different views of the same gob using some catadioptric elements ${ }^{6}$ to have as much information as possible to compensate any asymmetry. Experimentally, we found that the revolution symmetry of the gob is so high that using two views is redundant. A single view image is displayed in Fig. 2. Some dark spots appear on the object, due to shear lubricant droplets, and some bright areas appear on the background due to specular reflections on some shiny metallic feeder parts.

In the field of subpixel estimation, it is known that the accuracy of algorithms depends on the camera orientation with respect to the boundaries of objects. ${ }^{3-5}$ It is recommended to place the camera so that its reference frame axes intercept the longitudinal axis of the object at 45 deg to reduce the sampling pixel errors. If we would align the axis of the camera to the longitudinal axis of the gob, and consider a typical gob length of approximately 1000 pixels, the digitization error might be in the worst case $\pm 0.5 \%( \pm 2 \mathrm{~g})$. In our application, the camera is placed so that gobs always fall down with an angle between 1 and $3 \mathrm{deg}$. This slight rotation is enough to reduce significantly sampling errors down to $\pm 0.2 \mathrm{~g}$, which is reasonable.

\subsection{Segmentation and Improvement of the Image}

Before we can estimate the weight of the gob, it is necessary to segment the image, i.e., remove the background and extract the region of the image corresponding only to the gob. This segmentation task is usually done, especially for images with a high contrast, by binarization and requires the selection of a gray-level threshold. In Ref. 7, different techniques are described to select the threshold that involve choosing a gray level $t$, such all gray levels greater than $t$ are mapped into the object and the rest are mapped into background. When $t$ does not depend on the gray level of $(x, y)$ it is called global threshold and has the same value for all image points.
Plotting the histogram for a typical gob image (Fig. 2), we see that there are three peaks, the two of them to the left correspond to background and the one to the right is the gob. We have seen that the optimum gray-level value to be used as threshold, which is computed using classical methods based on assuming that a histogram is formed by two overlapped Gaussian distributions, ${ }^{7}$ performs worse than using a value selected experimentally. A threshold value of about 50 , in an 8-bit intensity gray scale ( 0 is black and 255 is white), is a good choice for the type of images processed in our application. Once the image is binarized [Fig. 2(a)] we see that some dark spots and bright areas are still present. Bright areas [Fig. 2(b)] have a typical size of around 150 pixels, which can affect the final gob weight estimation by $0.46 \mathrm{~g}$. The dark spots [Fig. 2(c)] are different in size and their contribution to the volume depends on whether they are in the edge or inside the object. Those inside the gob region have an area of about 220 pixels $(0.7$ g), but those in the boundary [Fig. 2(d)] are 350 pixel or higher $(1.08 \mathrm{~g})$.

To obtain an image without bright areas, a regionlabeling process is executed. Once the image is labeled, we connect the regions belonging to the same object but with different labels. Now that we have a gob with no bright areas but with dark spots, we eliminate them using a morphological operator (closing filter) that concatenates two actions: dilation and erosion. ${ }^{8}$ The dilatation function adds pixels to the boundaries of binary objects. This function will add pixels wherever pixels in the image intersect nonzero elements in the structuring element. The erode function removes pixels from binary objects in the same way that the dilation adds them. When the regions are expanded, the dark spots inside the gob disappear, and after erosion spots are not regenerated. Occasionally, large holes close to the boundary will remain unfiltered.

\subsection{Algorithms to Obtain the Weight}

Now that we have a segmented image free of noise, we want an estimation of the gob weight. Assuming that gobs can be modeled as ellipsoids (see Fig. 1) because they have symmetry of revolution around the major vertical axis $c$, and the gob horizontal cross section is approximately a circle defined by the minor axes $a$ and $b$, then the expression to compute the gob volume is volume $=4 / 3 \pi a b c$ $=4 / 3$ (area) $(b)$. The area is estimated by counting all pix- 


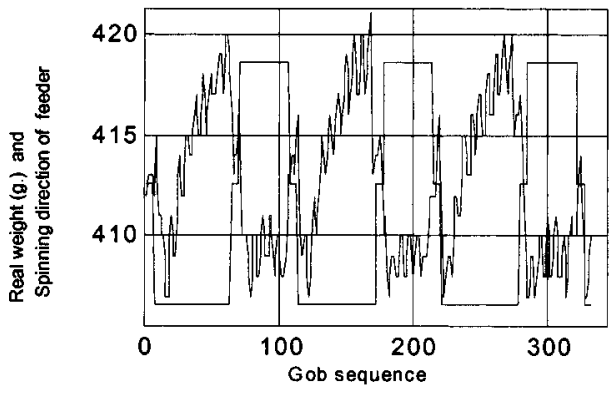

(a)

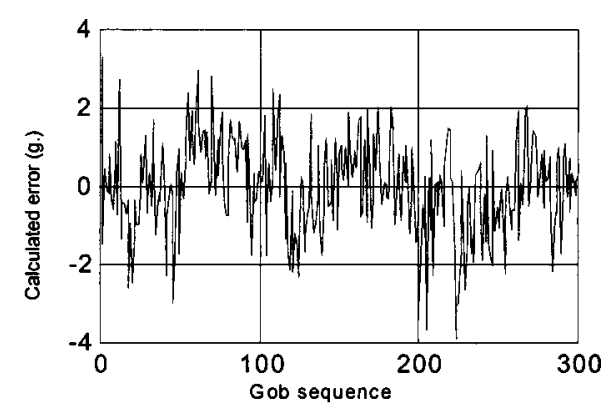

(b)

Fig. 3 (a) Real weight and CW (clockwise) and CCW (counterclockwise) tube rotations and (b) difference between calculated and real weights.

els set to one in the binary image. If we assume constant density, the weight of the gob is computed by this expression:

weight $=($ density $)($ volume $)=($ cte $)($ area $)(b)$.

Therefore the weight depends on a constant cte (including among others the glass density, focal length, etc.), the area of the gob in the image, and the semiminor axis of the ellipsoid $b$, which is not visible in the image. Note in Eq. (1) that the area and $b$ are dependent each other, so in fact weight does not depend linearly on area. Considering that the size and shape of gobs do not change significantly, we can assume that $b$ is quite stable and constant; therefore there is a linear relationship between weight and area:

weight $=\left(\right.$ cte $\left.^{\prime}\right)($ area $)$,

where cte' is a constant that depends on glass density, gob depth $(b)$, optical focal length, distance from camera to gob, and so on. This constant is estimated from the real weight value provided by the calibration module (Fig. 1 bottom), by a least-squares fitting.

\subsection{Experiments and Validation}

The validation of the described algorithms is experimentally done by means of capturing 300 gob images and weighing the corresponding final dishes. The reference value of this particular product is $410 \mathrm{~g}$. In Fig. 3(a) the real weights are plotted together with a representation of changes in the spinning direction of the feeder tube. We can see how those changes, represented by the square-like plot (top and bottom of square-like plot means CW and CCW spinning directions, respectively) affect the real weight. The difference between real weight and the values obtained with the image processing algorithms is depicted in Fig. 3(b), where we can observe that the maximum error in the weight measures is $\pm 3 \mathrm{~g}$. The root mean square (rms) value for the measurement algorithms is $1.2 \mathrm{~g}$.

\section{Control Subsystem}

This section presents the description of the control module designed for weight regulation and midterm disturbance cancellation. We present first the plant model identification procedure and then the design of a controller based on the previously identified plant model.

\subsection{Identification of the System Plant}

In our application, the plant to be controlled is the feeder, which we can consider as a single input single output (SISO) system where the input is the height of the feeder tube and the output is the weight of each gob. Other parameters such as glass temperature, tube rotation cycle, synchronization of shear, and so on, are considered as disturbances.

To obtain the plant model, we excited the feeder with a set of step-like tube displacements, while the output of the plant was registered. Using MATLAB's system identification toolbox and a sample period of $1 \mathrm{~s}$, we found that the discrete transfer function has four poles $(0.491 \pm 0.335 i$; $-0.33 \pm 0.31 i)$ and four zeros $(-1.52 ; 0.629 \pm 0.735 i ; 0)$ in the $z$ plane. One of the four zeros $(-1.52)$ is outside the unit circle, so the plant model is a non-minimum-phase system.

\subsection{Control Strategies}

In this paper, we have already described short-, mid-, and long-term disturbances, which correspond to weight noise of different types: random white noise, periodic noise, and drifts, respectively. Figure 4(a) illustrates short- and midterm disturbances for the open-loop plant (the measured rms disturbance is $10.1 \mathrm{~g}$ ).

The control strategy we present aims to cancel the midand long-terms disturbances and does not cope with the random component, which can be diminished only by improving the feeder design.

As we can see in Fig. 4(b), we have developed a proportional-integral (PI) controller to cancel long-term disturbances and some of the mid-term or periodic errors. The PI parameters $\left(K_{i}\right.$ and $\left.K_{p}\right)$ were selected to ensure stability under significant deviation between the plant model and the time-variant feeder plant. Those constants are $K_{i}=0.2$ and $K_{p}=0.2$. The PI controller eliminates longterm drifts and minimizes to some extent cyclic errors; the resulting rms error is $6.27 \mathrm{~g}$.

A learning control strategy using a repetitive controller (Fig. 5) is proposed, to improve the PI controller that is unable to cancel out all the cyclic disturbances. This type of control is especially appropriate for periodic disturbances, because it learns from previous cycles the control law to be generated in the future. Therefore, it is able to predict future perturbations anticipating the control action before the 

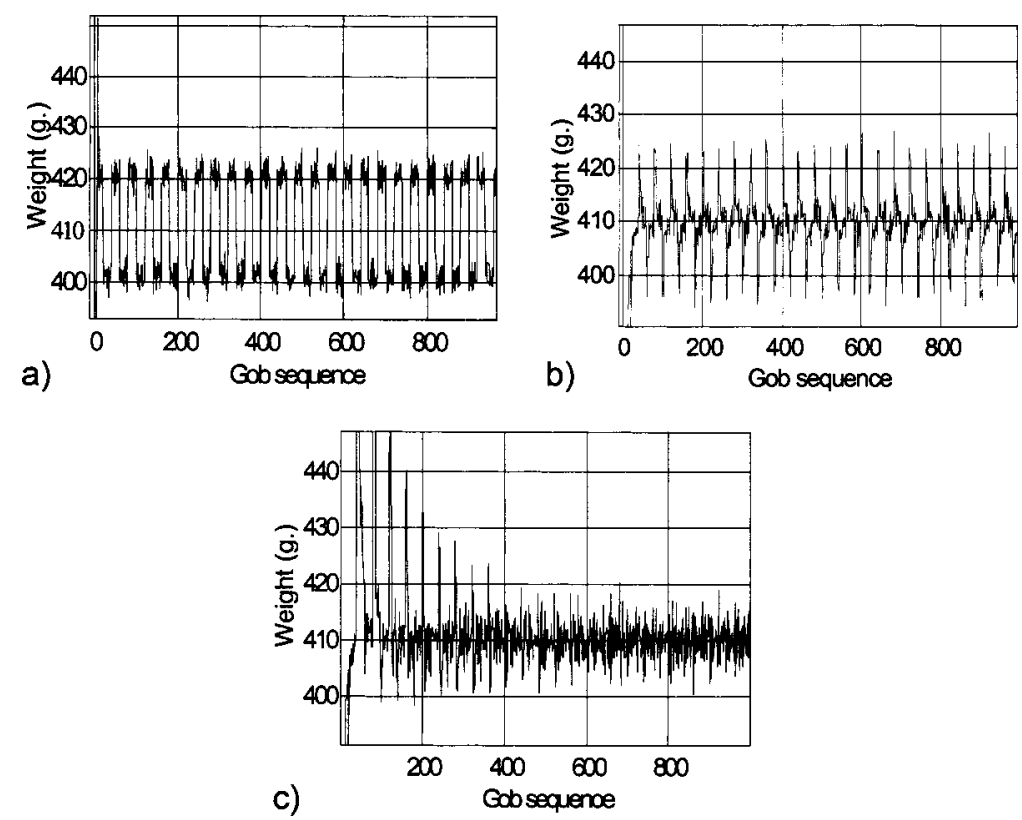

Fig. 4 (a) Open-loop response to a constant reference, (b) PI control performance, and (c) PI repetitive controls performance.

predicted disturbance appears. ${ }^{9-11}$ The repetitive controller is represented by this discrete equation

$\operatorname{out}_{R}(k)=\operatorname{out}_{R}(k-M)+K_{L}\left[\operatorname{in}_{R}(k-M)\right]$,

where $k$ is an integer value representing discrete time at the sampling period $T$ according to expression $t=k T$ ( $T$ is approximately $1 \mathrm{~s}$ ); $M$ is the period of the cyclic disturbance in units of samples (typically $M=40$ samples); $K_{L}$ is the learning constant that represents the speed of incorporating innovations into the learned sequence $\left(K_{L}=0.5\right)$; in $_{R}$ is the input to the repetitive controller, which corresponds to the weight error (Fig. 5, left); and out ${ }_{R}$ is the output of the repetitive controller that added to the reference weight is used as the reference to the PI closed-loop block (Fig. 5, right).

A zero-phase low-pass filter $F$ (see Fig. 5) is used inside the repetitive loop to make the repetitive controller more robust against nonperfect periodic disturbances. This filter makes the controller less effective in case of periodic disturbances, so a trade-off between robustness and effectiveness is found. The $z$-transform of the selected filter is in discrete time:
$\operatorname{out}_{F}(k)=(1 / 6) \operatorname{in}_{F}(k+1)+(2 / 3) \operatorname{in}_{F}(k)+(1 / 6) \operatorname{in}_{F}(k-1)$,

where $k$ is again the discrete time, $\mathrm{in}_{F}$ is the input to the $F$ filter, and out $F$ is the output of this filter.

The PI-repetitive control, as is show in Fig. 4(c), improves the response of the PI controller by itself, reducing most of the cyclic errors. The rms value, $3.6 \mathrm{~g}$, represents about a $40 \%$ error reduction compared to the classical PI controller.

\section{Conclusions}

The most common sources of weight variation in the process of fabrication of glass plates were analyzed. An algorithm for weight control of the forming glass gobs has been introduced, and it is outlined as follows. As a first step we developed a module for measuring the weight of molten glass gobs based on image-processing algorithms for estimating the area of these gobs. The segmented images were obtained by binarizing the acquired intensity images by a fixed threshold. Image noise was reduced using erosion and dilatation morphological operators and applying region la-

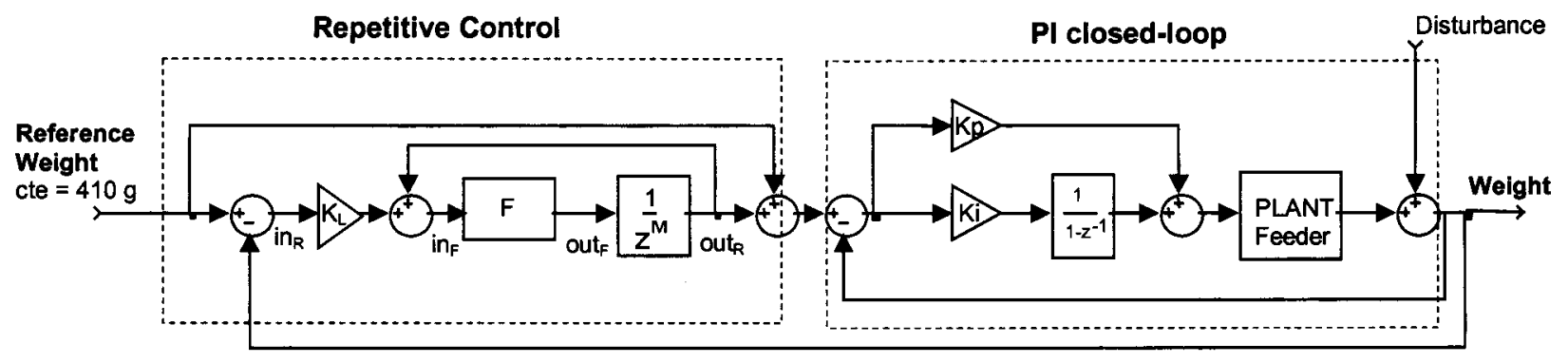

Fig. 5 PI-repetitive control diagram. 
beling and connection techniques. The estimation of the gob weight, which was derived from the area of the gob, was fed into the control loop. Typical weight estimation has a rms error of $1.2 \mathrm{~g}( \pm 3 \mathrm{~g})$, which for a usual product weight, accounts for only a $\pm 0.75 \%$ deviation.

Using the estimated gob weight and the reference value of each product, a learning repetitive control scheme is implemented that anticipates perturbations to reject disturbances. This control has a PI regulator to cancel long-term errors and some of the mid-term cyclic errors. A repetitive regulator was implemented to reject the periodic disturbances that are not totally cancelled by the classical PI control. A filter was added to give robustness against nonperiodic disturbances. Implementing a joint PI-repetitive control, we were able to keep the reference weight within $\pm 6 \mathrm{~g}$ (rms of $3.6 \mathrm{~g}$, about $\pm 1.5 \%$ deviation) and to cancel to a large extent the cyclic weight variations. Experimental results indicated that the measurement module and the control system perform satisfactorily, fitting expected quality requirements.

\section{References}

1. G. Ter Laak, "Continuous monitoring and control of the glass contribute to efficiency and high product quality," Int. Glass J. 93, 32-34 (1997).

2. R. Pascal and B. Barron, "Gob temperature control improves glass forming," Glass 52-53 (1998).

3. X. Liu and R. W. Ehrich, "Sub-pixel edge location in binary images using dithering," IEEE Trans. Pattern Anal. Mach. Intell. 17(6), 629634 (1995).

4. Y. Shan and G. W. Boon, "Sub-pixel location of edges with nonuniform blurring: a finite closed-form approach," Image Vis. Comput. 18, 1015-1023 (2000)

5. L. O'Gorman, "Sub-pixel precision of straight-edged shapes for registration and measurement," IEEE Trans. Pattern Anal. Mach. Intell. 18(7), 746-751 (1996).

6. J. Gluckman, "Rectified catadioptric stereo sensors," IEEE Trans. Pattern Anal. Mach. Intell. 4(2), 224-236 (2002).

7. J. S. Weszka, "A survey of threshold selection techniques," Comput Graph. Image Process. 7, 259-265 (1978).

8. K. Zhou and D. Wang, "Digital repetitive learning controller for three-phase CVCF PWM inverter," IEEE Trans. Ind. Electron. 48(4), $820-830$ (2001)

9. R. C. González and R. E. Woods, Tratamiento Digital de Imágenes, Addison-Wesley/Díaz de Santos, Reading, MA (1996).

10. G. Pajares and J. M. de la Cruz, Vision por Computador, Ra-Ma, Madrid (2001).

11. H. Rodriguez, J. L. Pons, and R. Ceres, "A ZPET-repetitive speed controller for ultrasonic motors," in Proc. IEEE Int. Conf. on Robotics and Automation, pp. 3654-3659 (2000).

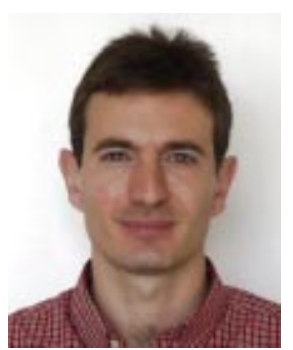

Antonio Ramón Jiménez Ruiz graduated in physics from the Computer Science Branch of the Universidad Complutense de Madrid in June 1991 and received his $\mathrm{PhD}$ degree, also in physics, from the Universidad Complutense de Madrid in October 1998. From 1991 to 1993 he worked in industrial laser applications at CETEMA (Technological Center of Madrid), Spain. Since 1994 he has been a researcher with the Instituto de Automática Industrial, CSIC, Spain. His current research interests include advanced sen- sory and processing technologies for localization, tracking, and extracting features of objects in sectors such as robotics, vehicle guiding, inspection, and machine tools.

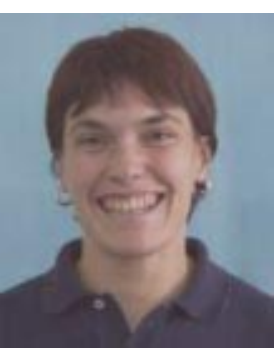

Eskarne Laizola Loinaz received her degree in physics from the Universidad de Pais Vasco in Leiona in 2000 and is currently working toward her PhD degree in science at the Instituto de Automática Industrial (IAI).

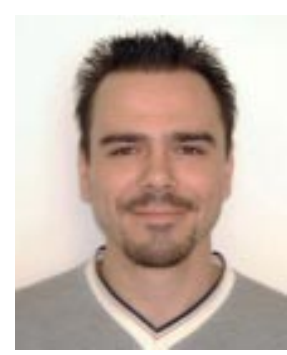

Fernando Morgado Rodríguez received his degree in electronics from the Universidad de Extremadura, Badajoz, in 2001 and is currently working toward his MS degree in information technologies at the Instituto de Automática Industrial (IAI).

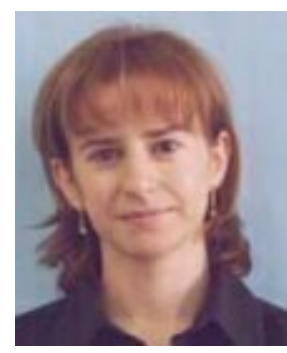

Mar Calvache Sánchez received her degree in electronics from the Universidad Complutense in Madrid in 2001 and is currently working toward her $\mathrm{PhD}$ degree in science at the Instituto de Automática Industrial (IAI).

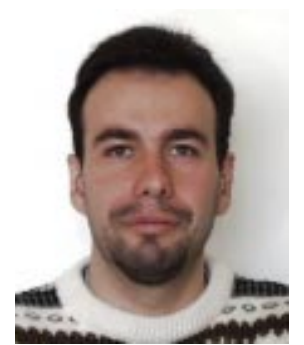

Fernando Seco Granja received his degree in physics from the Universidad Complutense in Madrid in 1996 and his PhD, also in physics, from the Universidad $\mathrm{Na}$ cional de Educación a Distancia in 2002. His dissertation dealt with the development of a linear position sensor based on the transmission of ultrasonic signals generated electromagnetically. Since 1997 he has been doing research at the Instituto de Automática Industrial (IAI). His interests include the electromagnetic generation of mechanical waves in metals, the propagation of sound in waveguides, the use of ultrasound in positioning, and the processing of ultrasonic signals. 